\title{
A novel finite element model for vibration analysis of rotating tapered Timoshenko beam of equal strength
}

\author{
Bulent Yardimoglu* \\ Department of Mechanical Engineering, Izmir Institute of Technology, 35430 Urla, Izmir, Turkey
}

\section{A R T I C L E I N F O}

\section{Article history:}

Received 19 May 2009

Accepted 23 May 2010

Available online 8 June 2010

\section{Keywords:}

Timoshenko beam

Finite element

Rotating beam

Equal strength

\begin{abstract}
A B S T R A C T
A new finite element model based on the coupled displacement field and the tapering functions of the beam is formulated for transverse vibrations of rotating Timoshenko beams of equal strength. In the coupled displacement field, the polynomial coefficients of transverse displacement and cross-sectional rotation are coupled through consideration of the differential equations of equilibrium. The tapering functions of breadth and depth of the beam are obtained from the principle of equal strength in the longitudinal direction of the beam. After finding the displacement functions using the tapering functions, the stiffness and mass matrices are expressed by using the strain and kinetic energy equations. A semi-symbolic computer program in Mathematica is developed and subsequently used to evaluate the new model. The results of the illustrative example regarding the problem indicated in the title of this paper are obtained and compared with the results found from the models created in ABAQUS. Very good agreement is found between the results of new model and the other results.
\end{abstract}

(c) 2010 Elsevier B.V. All rights reserved.

\section{Introduction}

The effects of shear deformation and rotary inertia are taken into account in Timoshenko beam theory [1] which is expressed by two coupled partial differential equations. For vibration of tapered beams, the aforementioned two coupled equations have variable coefficients. Furthermore, consideration of the rotational effects on the beam vibrations causes addition of a term with variable coefficient in these equations. Therefore, to find the exact solutions of these equations is generally difficult. Selecting a number of cross-section variation functions, the problem has been solved mainly by numerical or approximate methods such as the dynamic discretization technique [2], the spline interpolation technique [3], the transfer matrix method [4], and the method of Frobenius [5]. A carefully selected sample of the literature on the finite element studies for uniform and tapered rotating/nonrotating Timoshenko beams are given in author's previous study [6] which reports that there is no available shape functions incorporated with the taper parameters based on the breadth and/or depth of the tapered beam. Yardimoglu [6] derived the shape functions, considering the functions of breadth and depth of the tapered beam as power function for vibration analysis of rotating Timoshenko beam.

Hence, the objective of this paper is to present a novel finite element model based on the coupled displacement field incorporat-

\footnotetext{
* Tel.: +90 2327506781 .

E-mail addresses: bulentyardimoglu@iyte.edu.tr, bulyar@hotmail.com.
}

ing the taper functions of breadth and depth of the Timoshenko beam of equal strength in the longitudinal direction of the beam. The new finite element model has exact stiffness matrix, but approximate mass matrix owing to the usage of static equilibrium condition as in Refs. [7,8]. Petyt [9] reported that the usage of the static equilibrium condition in obtaining an approximate solution for the dynamic response requires an increase in the number of elements needed for a desired accuracy, but this is fully compensated by the simplicity of the mathematical analysis it provides. The new model is verified for out-of-plane vibration of rotating tapered Timoshenko beam of equal strength by comparison of the results obtained from the semi-symbolic computer program developed in Mathematica with the results found from the finite element models created in ABAQUS. In order to show the accuracy of the present new model clearly, the results are given in tabular form.

\section{Formulation of the breadth and depth of the beam}

Governing equation of equilibrium of a beam under distributed axial load $q(z)$ is given by Bickford [10] as

$\frac{d}{d z}[A(z) \sigma(z)]+q(z)=0$

The notation used throughout this paper is listed in the Nomenclature. For a rotating beam with constant angular velocity $\Omega$, distributed axial load is written as

$q(z)=\rho A(z) \Omega^{2} z$ 


\section{Nomenclature}

$A(z), A_{0}$ cross-sectional area of the beam and its coefficient

$b(z), b_{0}$ breadth of the beam and its coefficient

$[B]$ polynomial coefficients coupling matrix

$\left\{c_{v}\right\}$ polynomial coefficient vector of transverse displacement

$\left\{c_{\theta}\right\}$ polynomial coefficient vector of cross-sectional rotation

[C] element displacement-polynomial coefficient matrix

$C_{1}, C_{2} \quad$ constants of integration

$\operatorname{erfi}(z) \quad$ imaginary error function of $z$

$E, G \quad$ elastic modulus and shear modulus, respectively

$h(z), h_{0} \quad$ depth of the beam and its coefficient

$I(z), I_{x x 0}$ area moments of inertia of the beam cross-section about $x$ and its coefficient

$k \quad$ shear coefficient

$[K] \quad$ global elastic stiffness matrix

$\left[K_{e}\right] \quad$ element elastic stiffness matrix

$L \quad$ length of the beam

$m \quad$ breadth taper parameter

$M(z) \quad$ bending moment about $x$ axis

[M] global mass matrix

$\left[M_{e}\right] \quad$ element mass matrix

$n \quad$ depth taper parameter

$N \quad$ number of element

$p \quad$ taper parameter for area moments of inertia of the cross-section

$P(z)$
$\left[P_{v}\right]$

$\left[P_{\theta}\right]$

$q(z)$

$\{q\}$

$\left\{q_{e}\right\}$

$r$

$r_{g}$

SF

$[S]$

$\left[S_{e}\right]$

$T$

$U_{e}, U_{g} \quad$ elastic and geometric strain energies

$v(z, t) \quad$ transverse displacement

$v_{0}(t), v_{1}(t), v_{2}(t), v_{3}(t)$ polynomial coefficients of the transverse displacement

$V(z) \quad$ shear force in y direction

$z_{r} \quad$ co-ordinate of the root of the beam

$z_{t} \quad=z_{r}+L$ co-ordinate of the tip of the beam

$\theta(z, t) \quad$ cross-sectional rotation about $x$ axis

$\theta_{0}(t), \theta_{1}(t), \theta_{2}(t)$ polynomial coefficients of the cross-sectional rotation

$\sigma_{0} \quad=\sigma_{Y} / S F$, allowable stress

$\sigma_{Y} \quad$ yield stress

$\sigma(z) \quad$ normal stress

$\rho$ density

$\omega \quad$ natural circular frequency of beam

$\Omega \quad$ rotational speed
In order to obtain the cross-sectional area function of the beam of equal strength, normal stress $\sigma(z)$ in Eq. (1) is considered as constant, namely $\sigma(z)=\sigma_{0}$. Thus substituting Eq. (2) into Eq. (1), and then solving the equation yields,

$A(z)=A_{0} \exp \left(-r z^{2}\right)$

where

$r=0.5 \rho \Omega^{2} / \sigma_{0}$

Now, the breadth and depth of the beam depending on the parameters $m$ and $n$ which satisfy the relation $r=m+n$ are selected as follows:

$$
\begin{aligned}
& b(z)=b_{0} \exp \left(-m z^{2}\right) \\
& h(z)=h_{0} \exp \left(-n z^{2}\right)
\end{aligned}
$$

\section{Formulation of the finite element displacement functions}

The differential equations of motion for a nonuniform Timoshenko beam are given in Ref. [11]. The homogeneous form of these equations are written as follows:

$\frac{d M(z)}{d z}+V(z)=0$

$\frac{d V(z)}{d z}=0$

where

$$
\begin{aligned}
& M(z)=E I(z) \frac{d \theta(z)}{d z} \\
& V(z)=k A(z) G\left[\frac{d v}{d z}-\theta(z)\right]
\end{aligned}
$$

Considering the constant shear force given by Eq. (8) in Eq. (7), bending moment is obtained by integration as

$M(z)=C_{1} z+C_{2}$

The cross-sectional rotation of the beam is found by substituting Eq. (11) into Eq. (9), and integrating as

$\theta(z)=\int \frac{1}{\operatorname{EI}(z)}\left(C_{1} z+C_{2}\right) d z$

Transverse displacement of the beam is expressed by substituting Eq. (12) along with Eqs. (9) and (10) into Eq. (7), and integrating as

$v(z)=\int\left\{\theta(z)-\frac{1}{k A(z) G} \frac{d}{d z}\left[E I(z) \frac{d \theta(z)}{d z}\right]\right\} d z$

In order to express the displacement functions depending on the functions of the breadth and depth of the beam formulated in Eqs. (5) and (6), the area moment of inertia of the cross-section about $x$ axis can be written as

$I(z)=I_{x x 0} \exp \left(-p z^{2}\right)$

where $p=m+3 n$. Then, substituting Eq. (14) into Eq. (12), and integrating yields

$\theta(z, t)=\theta_{0}(t)+\theta_{1}(t) f_{1}(z)+\theta_{2}(t) f_{2}(z)$

where

$f_{1}(z)=\exp \left(-p z^{2}\right)$

$f_{2}(z)=\operatorname{erfi}(\sqrt{p} z)$

Also, substituting Eqs. (3), (14), and (15) into Eq. (13), and integrating yields

$v(z, t)=v_{0}(t)+v_{1}(t) g_{1}(z)+v_{2}(t) g_{2}(z)+v_{3}(t) g_{3}(z)$ 
where

$v_{1}(t)=\theta_{0}(t)$

$g_{1}(z)=z$

$v_{2}(t)=\theta_{1}(t)$

$g_{2}(z)=\frac{\sqrt{\pi} \operatorname{erfi}(\sqrt{p} z)}{2 \sqrt{p}}-\frac{\sqrt{\pi} p d I_{x x r} \operatorname{erfi}(\sqrt{r} z)}{A_{r} \sqrt{r}}$

$v_{3}(t)=\theta_{2}(t)$

$g_{3}(z)=-\frac{\exp \left(-p z^{2}\right)}{\sqrt{\pi p}}+z \operatorname{erfi}(\sqrt{p} z)$

The relationships between the coefficients of the crosssectional rotation and of the transverse displacement is written in the matrix form as

$\left\{c_{\theta}\right\}=[B]\left\{C_{v}\right\}$

where

$\left\{c_{\theta}\right\}=\left\{\theta_{0}(t) \quad \theta_{1}(t) \quad \theta_{2}(t)\right\}^{T}$

$\left\{c_{v}\right\}=\left\{\begin{array}{llll}v_{0}(t) & v_{1}(t) & v_{2}(t) & v_{3}(t)\end{array}\right\}^{T}$

$[B]=\left[\begin{array}{llll}0 & 1 & 0 & 0 \\ 0 & 0 & 1 & 0 \\ 0 & 0 & 0 & 1\end{array}\right]$

The $[B]$ matrix is termed as polynomial coefficients coupling matrix. For finite element formulation, the cross-sectional rotation given in Eq. (15) may be written as follows:

$\theta(z, t)=\left[P_{\theta}\right]\left\{c_{\theta}\right\}$

where

$\left[P_{\theta}\right]=\left[\begin{array}{lll}1 & f_{1}(z) & f_{2}(z)\end{array}\right]$

Also, the transverse displacement given in Eq. (17) may be expressed as follows:

$v(z, t)=\left[P_{v}\right]\left\{c_{v}\right\}$

where

$\left[P_{v}\right]=\left[\begin{array}{llll}1 & g_{1}(z) & g_{2}(z) & g_{3}(z)\end{array}\right]$

The element displacement vector of the finite element shown in Fig. 1 is written as

$\left\{q_{e}\right\}=\left\{\begin{array}{llll}v_{I} & \theta_{I} & v_{I I} & \theta_{I I}\end{array}\right\}^{T}$

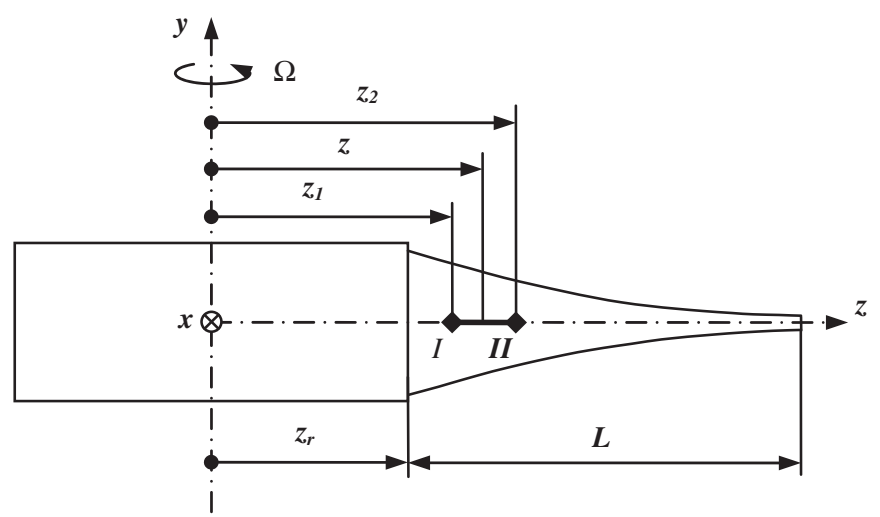

Fig. 1. Finite element model of rotating tapered Timoshenko beam.
On the other hand, the element displacement vector given in Eq. (27) can be expressed in terms of the polynomial coefficient vector of transverse displacement by using Eqs. (15), (17), and (18a), (18c), (18e) as follows:

$\left\{q_{e}\right\}=[C]\left\{c_{v}\right\}$

where

$[C]=\left[\begin{array}{llll}1 & g_{1}\left(z_{1}\right) & g_{2}\left(z_{1}\right) & g_{3}\left(z_{1}\right) \\ 0 & 1 & f_{1}\left(z_{1}\right) & f_{2}\left(z_{1}\right) \\ 1 & g_{1}\left(z_{2}\right) & g_{2}\left(z_{2}\right) & g_{3}\left(z_{2}\right) \\ 0 & 1 & f_{1}\left(z_{2}\right) & f_{2}\left(z_{2}\right)\end{array}\right]$

The matrix $[C]$ is termed as "element displacement-polynomial coefficient matrix”. Finally, Eq. (23) can be expressed by using Eqs. (19) and (28) as

$\theta(z, t)=\left[P_{\theta}\right][B][C]^{-1}\left\{q_{e}\right\}$

and also, Eq. (25) can be expressed by using Eq. (28) as

$v(z, t)=\left[P_{v}\right][C]^{-1}\left\{q_{e}\right\}$

\section{Derivation of the finite element mass and stiffness matrices}

The elastic strain energy of the Timoshenko beam is given in Refs. $[9,11]$ by

$U_{e}=0.5 \int_{z_{1}}^{z_{2}} \operatorname{EI}(z)\left(\frac{d \theta(z, t)}{d z}\right)^{2} d z+0.5 \int_{z_{1}}^{z_{2}} k G A(z)\left(\frac{d v(z, t)}{d z}-\theta(z, t)\right)^{2} d z$

The substitution of Eqs. (3), (14), (30), and (31) into Eq. (32) result in

$U_{e}=0.5\left\{q_{e}\right\}^{T}\left[K_{e}\right]\left\{q_{e}\right\}$

where $\left[K_{e}\right]$ is element elastic stiffness matrix given in Appendix A. The geometric strain energy due to centrifugal force $P(z)$ is given as follows [11]:

$U_{g}=0.5 \int_{z_{1}}^{z_{2}} P(z)\left(\frac{d v(z, t)}{d z}\right)^{2} d z$

where

$P(z)=\int_{z}^{z_{r}+L} q(z) d z$

Substituting Eq. (35) along with Eqs. (2), (3), and (31) into Eq. (34) gives

$U_{g}=0.5\left\{q_{e}\right\}^{T}\left[S_{e}\right]\left\{q_{e}\right\}$

where $\left[S_{e}\right]$ is element geometric stiffness matrix given in Appendix A. Finally, the kinetic energy of the Timoshenko beam is given as follows $[9,11]$ :

$T=0.5 \int_{z_{1}}^{z_{2}} \rho A(z)\left(\frac{d v(z, t)}{d t}\right)^{2} d z+0.5 \int_{z_{1}}^{z_{2}} \rho I(z)\left(\frac{d \theta(z, t)}{d t}\right)^{2} d z$

Similarly, the substitution of Eqs. (3), (14), (30), and (31) into Eq. (37) result in

$T=0.5\left\{\dot{q}_{e}\right\}^{T}\left[M_{e}\right]\left\{\dot{q}_{e}\right\}$

where overdot shows differentiation with respect to time and $\left[M_{e}\right]$ is element mass matrix given in Appendix A.

\section{Natural frequencies of the beam}

Global elastic stiffness, geometric stiffness, and mass matrices of the complete beam are obtained by assembling the element matrices given in Eqs. (A1), (A3), and (A5) in the usual way, and 
applying the boundary conditions as clamped at $z_{r}$-free. Since the integrand appeared in Eqs. (A2), (A4), and (A6) have erfi(z) as a factor, it is not possible to integrate the aforementioned integrals directly. Hence, the power series expansion about the origin of each element is used to calculate the element matrices in the computer program developed in Mathematica. Then, the natural frequencies of the rotating tapered Timoshenko beam of equal strength are found by solving the general eigenvalue problem given below

$\left(([K]+[S])-\omega^{2}[M]\right)\{q\}=0$

\section{Numerical example and discussion}

In order to design a beam geometry for illustrative example, geometrical properties of the beam must be found considering the rotational speed $\Omega$ and allowable normal stress $\sigma_{0}$. This idea requires to express the normal stress along the beam. Hence, substituting Eq. (2) into Eq. (35), dividing by Eq. (3) and integrating yields

$\sigma(z)=P(z) / A(z)=\sigma_{0}\left[1-\exp \left(r\left(z^{2}-z_{t}^{2}\right)\right)\right]$

where $r$ is given by Eq. (4). It is remarked just after Eq. (2) that $\sigma(z)=\sigma_{0}$ is considered, so Eq. (40) reduces to

$\exp \left(r\left(z^{2}-z_{t}^{2}\right)\right)=0$

Eq. (41) is satisfied only for the negative infinite value of the power of $e$, but this is physically and geometrically impossible. Geometric interpretation of Eq. (40) is shown in Fig. 2 for the reasonable values of taper parameter $r$. Considering the safety factor $S F=3$, the values of $r: 40,80$, and 160 appeared in Fig. 2 correspond to rotational speeds $\Omega: 1151.02 \mathrm{rad} / \mathrm{s}(10991.4 \mathrm{rpm})$, $1627.78 \mathrm{rad} / \mathrm{s}(15544.2 \mathrm{rpm})$, and $2302.03 \mathrm{rad} / \mathrm{s}(21982.8 \mathrm{rpm})$, respectively, for the material AISI-C 1040 of which $\rho=7850 \mathrm{~kg} / \mathrm{m}^{3}$ and $\sigma_{Y}=390 \mathrm{MPa}$ [12]. It can be seen from Fig. 2 that when $r$ increases, normal stress distribution along the beam approaches to $\sigma_{0}=130 \mathrm{MPa}$ for a larger interval of the beam.

From the foregoing discussion, the taper parameter $r$ forms the shape of the beam depending on the rotational speed $\Omega$ and allowable normal stress $\sigma_{0}$. On the other hand, the coefficients of breadth or depth of the beam ( $b_{0}$ and $h_{0}$ appeared in Eqs. (5) and (6), respectively) is determined by slenderness ratio.

As a numerical example, a tapered beam model based on the physical and geometrical parameters given in Table 1 is considered to illustrate the validity and accuracy of the new finite element model. In the computer program developed in Mathematica, the power series expansions of order 4 are employed. The convergence pattern of the new finite element for the aforementioned problem

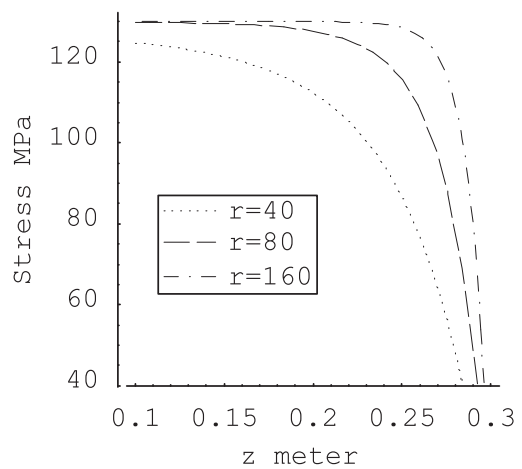

Fig. 2. Normal stress distribution along the beam for various taper parameters.
Table 1

Convergence pattern and comparison of natural frequencies (AISI-C 1040 [12] $\left.E / G=2.6, k=0.85, \sigma_{0}=130 \mathrm{MPa}, L=0.2 \mathrm{~m}, r_{g} / L=0.1, z_{r} / L=0.5, m=n=40, b_{0}=h_{0}\right)$.

\begin{tabular}{llcc}
\hline Natural frequencies $(\mathrm{Hz})$ & First & Second & Third \\
\hline Present $N=8$ & 2515.01 & 4498.27 & 6592.62 \\
Present $N=10$ & 2518.65 & 4526.98 & 7185.13 \\
Present $N=12$ & 2519.92 & 4536.01 & 7325.17 \\
Present $N=14$ & 2520.38 & 4539.12 & 7360.14 \\
Present $N=16$ & 2520.54 & 4540.23 & 7371.47 \\
Present $N=18$ & 2520.60 & 4540.62 & 7375.60 \\
Present $N=20$ & 2520.61 & 4540.71 & 7377.12 \\
Abaqus for C3D20R & 2513.80 & 4557.60 & 7453.60 \\
Abaqus for C3D20 & 2515.70 & 4565.10 & 7478.90 \\
\% Difference for C3D20R & 0.270905 & -0.37059 & -1.02608 \\
\% Difference for C3D20 & 0.195174 & -0.534271 & -1.3609 \\
\hline
\end{tabular}

and comparison of the present results with the results found from the model created in ABAQUS/CAE Student Edition 6.5-3 by using $3 \times 3 \times 17=153$ C3D20R elements (20-node hexahedral elements with reduced integration) and C3D20 elements (20-node hexahedral elements) are presented in Table 1 . The \% differences between of the present finite element results by using 20 elements and the results of Abaqus (\% Difference $=100$ [Present/Abaqus - 1]) are also given in Table 1 . It is evident that the results presented in Table 1 are very consistent.

\section{Conclusion}

A new finite element model based on the coupled displacement field has been developed for the vibration analysis of rotating tapered Timoshenko beam of equal strength in the longitudinal direction of the beam. The functions of breadth and depth of the beam of equal strength have been taken into account in the derivation of the displacement functions of the finite element model. A semi-symbolic computer program in Mathematica is developed and then used to evaluate the new model. The convergency, accuracy, and efficiency of the new model have been demonstrated by means of an illustrative example. Rapid convergence has been observed in the present model results. Moreover, the present results are in very good agreement with the results obtained by the model created in ABAQUS.

\section{Appendix A. Finite element matrices}

$\left[K_{e}\right]=[C]^{-T}[k][C]^{-1}$

where

$$
\begin{aligned}
{[k] } & =[B]^{T}\left\{E \int_{z_{1}}^{z_{2}} I(z)\left[P_{\theta}^{\prime}\right]^{T}\left[P_{\theta}^{\prime}\right] d z+k G \int_{z_{1}}^{z_{2}} A(z)\left[P_{\theta}\right]^{T}\left[P_{\theta}\right] d z\right\}[B] \\
& -k G\left\{[B]^{T} \int_{z_{1}}^{z_{2}} A(z)\left[P_{\theta}\right]^{T}\left[P_{v}^{\prime}\right] d z+\int_{z_{1}}^{z_{2}} A(z)\left[P_{v}^{\prime}\right]^{T}\left[P_{\theta}\right] d z[B]\right\} \\
& +k G \int_{z_{1}}^{z_{2}} A(z)\left[P_{v}^{\prime}\right]^{T}\left[P_{v}^{\prime}\right] d z
\end{aligned}
$$

Throughout these equations, prime represents differentiation with respect to $z$.

$\left[S_{e}\right]=[C]^{-T}[S][C]^{-1}$

where

$[s]=\int_{z_{1}}^{z_{2}} P(z)\left[P_{v}^{\prime}\right]^{T}\left[P_{v}^{\prime}\right] d z$

$\left[M_{e}\right]=[C]^{-T}[m][C]^{-1}$ 
where

$[m]=\rho\left\{\int_{z_{1}}^{z_{2}} A(z)\left[P_{v}\right]^{T}\left[P_{v}\right] d z+[B]^{T} \int_{z_{1}}^{z_{2}} I(z)\left[P_{\theta}\right]^{T}\left[P_{\theta}\right] d z[B]\right\}$

\section{References}

[1] S.P. Timoshenko, On the correction for shear of the differential equation for transverse vibrations of prismatic bars, Philosophical Magazine 41 (1921) 744-746.

[2] B. Downs, Transverse vibrations of cantilever beams having unequal breadth and depth tapers, Journal of Applied Mechanics ASME 44 (1977) 737-742.

[3] T. Irie, G. Yamada, I. Takahashi, Determination of the steady state response of a Timoshenko beam of varying cross-section by use of the spline interpolation technique, Journal of Sound and Vibration 63 (2) (1979) 287-295.

[4] T. Irie, G. Yamada, I. Takahashi, Vibration and stability of a non-uniform Timoshenko beam subjected to a follower force, Journal of Sound and Vibration 70 (4) (1980) 503-512.
[5] S.Y. Lee, S.M. Lin, Exact vibration solutions for nonuniform Timoshenko beams with attachments, AIAA Journal 30 (12) (1992) 2930-2934.

[6] B. Yardimoglu, Vibration analysis of rotating tapered Timoshenko beams by a new finite element model, Shock and Vibration 13 (2006) 117-126.

[7] R. Davis, R.D. Henshell, G.B.A. Warburton, Timoshenko beam element, Journal of Sound and Vibration 22 (4) (1972) 475-487.

[8] D.J. Dawe, A finite element for the vibration analysis of Timoshenko beams Journal of Sound and Vibration 60 (1) (1978) 11-20.

[9] M. Petyt, Introduction to Finite Element Vibration Analysis, Cambridge University Press, Cambridge, 1990.

[10] W.B. Bickford, Advanced Mechanics of Materials, Addison-Wesley, California 1998.

[11] L. Meirovitch, Analytical Methods in Vibrations, Macmillan, New York, 1967.

[12] A.P. Boresi, R.J. Schmidt, O.M. Sidebottom, Advanced Mechanics of Materials, John-Wiley, New York, 1993. 